\title{
Periodic and Infinite Traces in Matrix Semigroups ${ }^{\star}$
}

\author{
Paul Bell ${ }^{1}$ and Igor Potapov ${ }^{2}$ \\ 1 Department of Mathematics, Turku University \\ paubel@utu.fi \\ 2 Department of Computer Science, University of Liverpool \\ potapov@liverpool.ac.uk
}

\begin{abstract}
In this paper we provide several new results concerning word and matrix semigroup problems using counter automaton models. As a main result, we prove a new version of Post's correspondence problem to be undecidable and show its application to matrix semigroup problems, such as Any Diagonal Matrix Problem and Recurrent Matrix Problem. We also use infinite periodic traces in counter automaton models to show the undecidability of a new variation of the Infinite Post Correspondence Problem and Vector Ambiguity Problem for matrix semigroups.
\end{abstract}

\section{Introduction}

Over the last few decades, there has been considerable interest in algorithmic problems for matrix semigroups. Matrices are commonly used objects in mathematics, physics, engineering and other areas of science. Many questions about the dynamics of finite or infinite matrix products tightly connect problems with computation theory and theoretical computer science in general.

It is known that semigroups generated by matrices have a number of undecidable problems. Therefore many questions related to processes with iterative matrix multiplication are very hard to analyse and answer in general.

The majority of the investigated questions related to matrix semigroups were about direct reachability: membership problems (can a particular matrix be constructed by multiplication of matrices from a given set of generators?); vector reachability problems (can one particular vector be reached from another one by means of a set of linear transformations from a given set of generators?) and scalar reachability problems and their variants $[2,3,8]$. The only problem that may stand alone is the freeness problem for matrix semigroups: "Does every matrix in the semigroup have a unique factorization?" [6]. What might be interesting in terms of further understanding of the fundamental properties of matrix semigroups is to analyse the behavioural properties of possible traces and their relations.

The undecidability results for matrix semigroups were mainly shown by a reduction from the undecidability of Post's Correspondence Problem (PCP).

In this paper we want to highlight a number of "cross references" and connections between matrix semigroup problems and behavioral properties of counter machines. As a main result, we show that the Vector Ambiguity Problem and Recurrent Matrix Problem are undecidable for finitely generated matrix semigroups over integer and rational numbers. We also show that

\footnotetext{
* This work was partially supported by Royal Society IJP 2007/R1 grant
} 
the problem of reaching any diagonal matrix is undecidable for $4 \times 4$ matrix semigroups over complex numbers. Moreover, using counter machine models, we provide a new undecidable variant of the infinite Post correspondence problem.

\section{Preliminaries}

In this section we introduce standard definitions and show some technical results about periodic traces in counter automata with their proofs to make the paper self contained.

Let us denote an empty word by $\varepsilon$. For two words $u=u_{1} u_{2} \cdots u_{m}$ and $v=v_{1} v_{2} \cdots v_{n}$ we denote their concatenation by $u \cdot v=u_{1} u_{2} \cdots u_{m} v_{1} v_{2} \cdots v_{n}$. For the inverse of a letter ' $a$ ' we sometimes write ' $\bar{a}$ '. Given a word $w=w_{1} w_{2} \cdots w_{k}$, we also define $\bar{w}=w^{-1}=\overline{w_{k}} \cdots \overline{w_{2}} \overline{w_{1}}$. We denote a word $\underbrace{a a \cdots a}_{k}$ by $a^{k}$.

Post's correspondence problem (PCP) is formulated as follows: Given a finite alphabet $\Sigma$ and a finite (ordered) set of pairs of words in $\Sigma^{*}:\left\{\left(u_{1}, v_{1}\right), \ldots,\left(u_{k}, v_{k}\right)\right\}$. Does there exist a finite sequence of indices $\left(i_{1}, i_{2}, \ldots, i_{m}\right)$ with $1 \leq i_{j} \leq k$ for $1 \leq j \leq m$, such that $u_{i_{1}} u_{i_{2}} \cdots u_{i_{m}}=v_{i_{1}} v_{i_{2}} \cdots v_{i_{m}}$. It can be shown that this problem is undecidable even with a binary alphabet $\Sigma$.

\subsection{Two-Counter Minsky Machines}

In this subsection we shall describe a well known computational model known as a Minsky machine. Informally speaking, a Minsky machine is a two-counter machine that can increment and decrement counters by one and test them for zero. It is known that Minsky machines are a universal model of computation [15]. Being of very simple structure, Minsky machines are very useful for proving undecidability results (see for example [11] or [12]).

It is convenient to represent a counter machine as a simple imperative program $\mathcal{M}$ consisting of a sequence of instructions labeled by natural numbers from 1 to some $L$. Any instruction is one of the following forms:

$l$ : ADD 1 to $S_{k}$; GOTO $l^{\prime}$;

$l:$ IF $S_{k} \neq 0$ THEN SUBTRACT 1 FROM $S_{k}$; GOTO $l^{\prime}$ ELSE GOTO $l^{\prime \prime}$;

$l$ : STOP.

where $k \in\{1,2\}$ and $l, l^{\prime}, l^{\prime \prime} \in\{1, \ldots, L\}$.

The machine $\mathcal{M}$ starts executing with initial nonnegative integer values in counters $S_{1}$ and $S_{2}$ and the control at instruction 1 . We assume the semantics of all the above instructions and of the entire program is clear. Without loss of generality, one can suppose that every machine contains exactly one instruction of the form $l$ : STOP which is the last one $(l=L)$. It should be clear that the execution process (run) is deterministic and has no failure. Any such process is either finished by the execution of instruction $L$ : STOP, or lasts forever.

As a consequence of the universality of such a computational model, the halting problem for Minsky machines is undecidable:

Proposition 1 ([15]). It is undecidable whether a two-counter Minsky machine halts when both counters initially contain 0 . 
In this paper, unless otherwise stated, we assume all machines start with 0 in both counters. Minsky machines can be simulated by PCP in a direct way. Given a two-counter machine $M$, our aim is to produce a set of pairs of words $P=\left\{\left(u_{1}, v_{1}\right),\left(u_{2}, v_{2}\right), \ldots,\left(u_{n}, v_{n}\right)\right\}$ such that there exists a finite sequence of indices $S=\left(i_{1}, i_{2}, \ldots, i_{k}\right)$ with each $1 \leq i_{j} \leq n$ where $u_{i_{1}} u_{i_{2}} \cdots u_{i_{k}}=v_{i_{1}} v_{i_{2}} \cdots v_{i_{k}}$ if and only if $M$ halts when starting from the initial state with 0 in both counters. The sequence $S$ we shall call a solution of the instance and it corresponds to a halting run of $M$ and can be seen as an instance of PCP. Another option is to consider an infinite sequence $S$ that corresponds to an infinite run of a counter machine.

Proposition 2. A two-counter automata CA can be simulated by Post's correspondence problem (PCP), where PCP has a solution if and only if $C A$ halts.

Proof. We use the definitions of a two-counter machine from [10]. We require two operations, firstly "from state $q$, increment counter $\{1,2\}$ and move to state $s$ ". Secondly, we require an operation to "test if counter $\{1,2\}$ is zero, moving to state $r$ if it is, or state $t$ if it is positive". We shall use the symbol ' $z$ ' throughout to denote a zero counter.

We start with an initial pair of words $\left(u_{1}, v_{1}\right)=\left(\#, \# z a^{i} q_{0} a^{j} z \#\right)$ where $i$ denotes the initial value of the counter $C_{1}$ and $j$ denotes the initial value of the counter $C_{2}$. Let us deal with the first type of operation. To move from state $q$ to $s$ and increment $C_{1}$, we add the pair $(q, a s)$ to $P$. To move from state $q$ to $s$ and increment $C_{2}$, we add the pair $(q, s a)$ to $P$. But the counters could be zero (denoted $z q C_{2}$ or $\left.C_{1} q z\right)$ so we also add pairs $(z q, z a s)$ to increment $C_{1}$ and $(q z, s a z)$ to increment $C_{2}$.

For the second operation, we require to move from $q$ to $r$ if $C_{1}$ is zero, so we add pair $(z q, z r)$. To move from $q$ to $r$ if $C_{2}$ is zero, we add pair $(q z, r z)$. To move from $q$ to $t$ and decrement $C_{1}$ if not zero, we add pair $(a q, t)$ and to move from $q$ to $t$ and decrement $C_{2}$ if not zero, we add pair $(q a, t)$. Finally, we add pairs $(a, a),(\#, \#),(z, z),\left(a q_{\text {accept }}, q_{\text {accept }}\right)$, $\left(q_{\text {accept }} a, q_{\text {accept }}\right)$ and $(\# \triangleright, \triangleright)$ to $P$ where $\triangleright$ is a new symbol.

We can enforce that the first pair used must be $\left(u_{1}, v_{1}\right)$ by using a word mapping. Let $y=y_{1} y_{2} \cdots y_{n} \in \Gamma^{*}$ be any word and let ' $*$ ' be a new letter not in $\Gamma$. Then define the three mappings:

$$
\begin{gathered}
\star y=\quad * y_{1} * y_{2} \cdots * y_{n} \\
y \star=y_{1} * y_{2} * \cdots y_{n} * \\
\star y \star=* y_{1} * y_{2} * \cdots * y_{n} *
\end{gathered}
$$

We apply one of the three above $\star$ mappings to each word pair. Let $\left(u_{1}, v_{1}\right)=\left(\star u_{1}, \star v_{1} \star\right)$, $\left(u_{j}, v_{j}\right)=\left(\star u_{j}, v_{j} \star\right)$ for each $2 \leq j \leq(n-1)$ and $\left(u_{n}, v_{n}\right)=(\# * \triangleright, \triangleright)$ also. Clearly if a match occurs in $P$ it must start with this new $\left(u_{1}, v_{1}\right)$ since only the first two letters in these two words are equal. Examining the mapping allows us to conclude it must then proceed as before using the new pairs $\left(u_{i}, v_{i}\right)$ with $2 \leq i \leq n$ and finally finish with the pair $\left(u_{n}, v_{n}\right)$, see [17] for further details since this is similar to how a Turing machine is encoded.

If there exists some sequence $S=\left(i_{1}, i_{2}, \ldots, i_{k}\right)$ such that $u_{1} u_{i_{1}} u_{i_{2}} \cdots u_{i_{k}}=v_{1} v_{i_{1}} v_{i_{2}} \cdots v_{i_{k}}$ then it corresponds to a correct halting computation of a two-counter machine and it is thus undecidable whether such a sequence $S$ exists.

\subsection{Periodicity in Counter Machines}

It was proven in [7] that given a counter machine $M$, we can construct a second counter machine, $M^{\prime}$, such that $M^{\prime}$ never halts and has a periodic configuration if and only if $M$ halts. 
Since the halting problem for arbitrary two-counter machines is undecidable, this means that checking the periodicity is also undecidable. We shall now give a simple proof to the above result from [7] for completeness:

Proposition 3. Let $M^{\prime}$ be a counter machine that has no halting configuration. The problem of deciding if $M^{\prime}$ has a periodic configuration is undecidable ${ }^{3}$.

Proof. Given a specific counter machine $M$. Let $q_{0}$ be the initial state of $M$ and $H=$ $\left\{q_{h_{1}}, q_{h_{2}}, \ldots, q_{h_{t}}\right\}$ be the set of halting states. Let $R=\left\{R_{1}, R_{2}, \ldots, R_{k}\right\}$ be the set of counters of $M$. See Figure 1 a.

a)

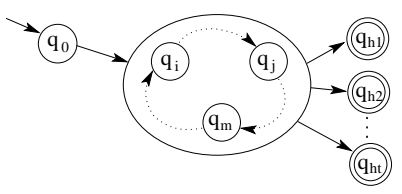

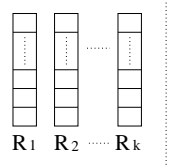

b)

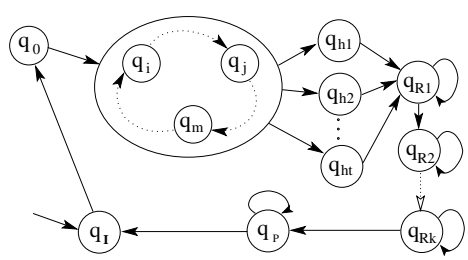

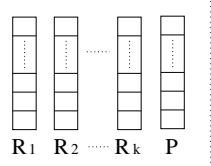

Fig. 1. a) Minsky machine with $k$ counters b) Periodic Minsky machine

We shall now show how to create a new machine $M^{\prime}$. Initially, let $M^{\prime}$ have the same states $Q$ as $M$ and the same transition function $\delta$. We add a new start state $q_{I}$ and add the two rules to $\delta$ which move from $q_{I}$ to state $q_{0}$ regardless of whether the first counter $R_{1}$ equals zero and leaves all counters as they are.

We define all states $q \in H$ to be non-halting states and add new states $q_{R_{1}}, q_{R_{2}}, \ldots, q_{R_{k}}$. These new states will be used to zero all counters. We add rules which move us from each $q \in H$ to $q_{R_{1}}$ regardless of whether $R_{1}$ is non-zero and leave all counters at their current values. Then for each state $q_{R_{i}}, 1 \leq i<k$ we add rules which decrease $R_{i}$ if it is non-zero and remain in the current state. We add a rule to move to state $q_{R_{i+1}}$ if it does equal zero (thus the counter is decremented to zero before going to the next state). Finally, for state $q_{R_{k}}$ we add a rule to decrease $R_{k}$ if it is non-zero and stay in state $q_{R_{k}}$, or else move to the initial state $q_{I}$ if it does equal zero (note that once we go to $q_{I}$, all counters are equal to 0 and we are back to the original configuration).

Thus, if $M$ reached a halting state with some values in its counters $R$, then $M^{\prime}$ will instead decrement all counters to zero and restart the computation. Clearly the only way to get back to $q_{I}$ is via some state in $H$ of $M$, thus the only way $M^{\prime}$ is periodic is if $M$ halts as required.

We may note that there may be some other configuration of $M$ which is periodic, thus $M^{\prime}$ will contain an ultimately periodic configuration (though not periodic since it still will not go to $\left.q_{I}\right)$. We can avoid this situation if required by a simple construction. Add a new counter $P$ such that every transition of machine $M$ increments $P$ and then does what it would do normally (we need to add new states and rules to do this). Then add a new state $q_{P}$ such that

\footnotetext{
${ }^{3}$ The problem is undecidable even in the case of two-counter machines
} 
$q_{R_{k}}$ now goes to $q_{P}$ instead of $q_{I}$. Then add rules to decrement $q_{P}$ to zero as before and then move to state $q_{I}$. The only way to decrement counter $P$ is via a halting state thus now the only periodic configurations contain $q_{I}$ with all counters zero in their period. See Figure $1 \mathrm{~b}$.

Proposition 4. Let $M^{\prime}$ be a nondeterministic n-counter machine. The problem of deciding if $M^{\prime}$ has an infinite number of trajectories leading to a halting state $q_{\text {final }}$ with zero in all counters is undecidable for any $n \geq 2$.

Proof. The problem of determining if a counter machine $M$ can reach a halting state with zero counters is undecidable. Without loss of generality we can also assume that the initial state of $M$ will be visited only once. Let us construct a new nondeterministic counter machine $M^{\prime}$ based on a deterministic counter machine $M$ as follows. First, we add two extra states $q_{\text {final }}$ (which is the only halting state of $M^{\prime}$ ) and $q_{\text {continue. Then add transitions from all halting }}$ states of $M$ leading to both $q_{\text {final }}$ and $q_{\text {continue }}$ which will only be executed if both counters are zero. Secondly, we create copies of all transitions from the inital state of $M$ and add them to the automaton as outgoing transitions from $q_{\text {continue }}$.

As a result, we have that the initial state of $M^{\prime}$ (which is the same as in $M$ ) will be visited only once and state $q_{\text {final }}$ with zero counters is reachable in $M^{\prime}$ if and only if machine $M$ can reach a halting state with zero counters. On the other hand, if $q_{\text {final }}$ with zero counters can be reached at least once, we can construct an infinite number of traces that will lead to $q_{\text {final }}$ by returning from the halting state of $M$ to $q_{\text {continue }}$ and repeating the same looping trace an unbounded number of times before going to state $q_{\text {final }}$.

\section{Fixed Element PCP}

Problem 1. Fixed Element PCP - Given an alphabet $\Gamma=\left\{a, b, a^{-1}, b^{-1}, \Delta, \Delta^{-1}, \star\right\}$ where $\Gamma \backslash\{\star\}$ forms a free group not containing ' $\star$ ', and a finite set of pairs of words over $\Gamma$,

$$
P=\left\{\left(u_{1}, v_{1}\right),\left(u_{2}, v_{2}\right), \ldots,\left(u_{n}, v_{n}\right)\right\} \subset \Gamma^{*} \times \Gamma^{*} .
$$

Does there exist a finite sequence of indices $s=\left(s_{1}, s_{2}, \ldots, s_{k}\right)$ such that $u_{s_{1}} u_{s_{2}} \cdots u_{s_{k}}=$ $v_{s_{1}} v_{s_{2}} \cdots v_{s_{k}}=\star ?$

This variant of PCP is interesting since it is similar to the standard PCP however instead of testing for a solution via equality checking for two arbitrary words, the solutions will have a specific form of a fixed letter $\star$.

Theorem 1. The Fixed Element PCP is undecidable.

Proof. The instance set of Fixed Element PCP will now be defined. Given an instance of PCP over $\Sigma=\{a, b\}$ where the first and last pairs are used exactly once ${ }^{4}$ :

$$
P^{\prime}=\left\{\left(u_{1}, v_{1}\right),\left(u_{2}, v_{2}\right), \ldots,\left(u_{m}, v_{m}\right)\right\} \subset \Sigma^{*} \times \Sigma^{*}
$$

we shall define two sets $L, R$ such that $P=L \cup R$ :

$$
\begin{array}{r}
L=\left\{\left(\star u_{1}, \star \bar{\Delta} b a b\right),\left(u_{i}, a^{i} b\right)\right\} \subset \Gamma^{*} \times \Gamma^{*} \quad ; 2 \leq i \leq m \\
R=\left\{\left(\bar{v}_{i}, \bar{\Delta} \bar{a}^{i} \bar{b} \Delta\right),\left(\overline{v_{m}}, \bar{b} \bar{a}^{m} \bar{b} \Delta\right)\right\} \subset \Gamma^{*} \times \Gamma^{*} \quad ; 1 \leq i \leq m-1
\end{array}
$$

\footnotetext{
${ }^{4}$ This is standard in proofs of undecidability of PCP, see the construction in [17]
} 
Since we are looking for a product of pairs of words equal to $(\star, \star)$ and $\nwarrow \notin \Gamma$, then the first pair $L_{1}=\left(\star u_{1}, \star \bar{\Delta} b a b\right)$ must occur exactly once. Let us denote any such product (if it exists) as:

$$
X=(\star, \star)=X_{1} X_{2} \cdots X_{k} \in\langle L \cup R\rangle .
$$

It can be seen that $X_{1}=L_{1}$, otherwise if $X_{j}=L_{1}$ for some $j>1$, then:

$$
\left\langle(L \cup R) \backslash\left\{L_{1}\right\}\right\rangle \ni X_{1} X_{2} \cdots X_{j-1}=(\varepsilon, \varepsilon),
$$

but this is impossible since clearly $\varepsilon$ is not in the subsemigroup generated by $\left\{\bar{b} \bar{a}^{m} \bar{b} \Delta, a^{i} b, \bar{\Delta} \bar{a}^{i} \bar{b} \Delta\right.$ : $1 \leq i \leq m-1\}$, therefore the second word cannot equal $\varepsilon$. This follows since the inverse of ' $\bar{b} \Delta$ ' clearly cannot be found as a prefix of any word in the subsemigroup and thus an element equal to $\varepsilon$ would have to begin using elements of the form $a^{i} b$. But this must eventually concatenate with $\bar{b} \bar{a}^{m} \bar{b} \Delta$ to reduce the word, and again we will have ' $\bar{b} \Delta$ ' on the right which then cannot be reduced. Therefore we must have:

$$
X=L_{1} X_{2} \cdots X_{k}=(\star, \star) .
$$

Let us consider the second words, in order to determine the sequence they must take to give ' $\star$ '. We have the set of elements:

$$
A=\left\{\star \bar{\Delta} b a b, \bar{b} \bar{a}^{m} \bar{b} \Delta, a^{2} b, \ldots, a^{m} b, \bar{\Delta} \bar{a} \bar{b} \Delta, \ldots, \bar{\Delta} \bar{a}^{m-1} \bar{b} \Delta\right\}
$$

We know the first element is $(\star \bar{\Delta} b a b)$ which is used only once. We now show that the only products equal to ' $\star$ ' are of the form:

$$
L_{1} L_{i_{1}} L_{i_{2}} \cdots L_{m} R_{m} \cdots R_{i_{2}} R_{i_{1}} R_{1}
$$

for some $i_{1}, i_{2}, \ldots, i_{l} \in\{2, \ldots, m-1\}$. Since $(\star \bar{\Delta} b a b)$ is the first element used, assume that the next element is from $R$, i.e., of the form $\left(\bar{\Delta}^{i} \bar{a}^{i} \Delta\right)$ for some $1 \leq i \leq m-1$ or $\left(\bar{b} \bar{a}^{m} \bar{b} \Delta\right)$. But this gives ' $(\star \bar{\Delta} b a b)\left(\bar{\Delta} \bar{a}^{i} \bar{b} \Delta\right)$ ' or ' $\left(\star \bar{\Delta} b \bar{a}^{m-1} \bar{b} \Delta\right)$ ' and both cannot be reduced by further right multiplications since clearly from the set $A$, there is not any product of elements with a $\bar{\Delta} b$ ' on the left hand side.

Thus, the only option is for the second element to be of the form $\left(a^{i} b\right)$ for $2 \leq i \leq m$. Let $j+1$ be the first index at which we do not have an element from $L$, thus the product $X_{1} X_{2} \cdots X_{j}$ is of the form: $(\star \bar{\Delta} b a b)\left(a^{i_{2}} b\right)\left(a^{i_{3}} b\right) \cdots\left(a^{i_{j}} b\right)$ where $2 \leq i_{2}, i_{3}, \ldots, i_{j} \leq m$. To reduce this product, the next element must be $\left(\bar{b} \bar{a}^{m} \bar{b} \Delta\right)$ since this is the only element with a ' $\bar{b}$ ' on the left. The product of $\left(a^{i_{j}} b\right)\left(\bar{b} \bar{a}^{m} \bar{b} \Delta\right)$ is $\bar{a}^{m-i_{j}} \bar{b} \Delta$. If $i_{j}<m$, then this will not reduce to ' $\bar{b} \Delta$ ' and so the left ' $\bar{b}$ ' will not cancel. Similarly to before, we cannot reduce this product any further since the right hand element is ' $\bar{b} \Delta$ '. Thus $i_{j}=m$.

The next element to the right cannot be $\left(\bar{b} \bar{a}^{m} \bar{b} \Delta\right)$ since the left hand letter does not cancel with $\Delta$ and it will have ' $\bar{b} \Delta$ ' on the right hand side which cannot be canceled. Similarly, the next element cannot be of the form $\left(a^{k_{1}} b\right)$ since this would then give:

$$
(\star \bar{\Delta} b a b)\left(a^{i_{2}} b\right)\left(a^{i_{3}} b\right) \cdots\left(a^{i_{j}}\right) \Delta a^{k_{1}} b,
$$

and again this is only reduced with $\left(\bar{b} \bar{a}^{m} \bar{b} \Delta\right)$, but

$$
\left(a^{i_{j}}\right) \Delta a^{k_{1}} b\left(\bar{b} \bar{a}^{m} \bar{b} \Delta\right)=a^{i_{j}} \Delta \bar{a}^{m-k_{1}} \bar{b} \Delta,
$$


and regardless of whether $k_{1}=m$, the product ends with ' $\bar{b} \Delta$ ' which cannot be reduced.

Thus, the next element must be of the form $\left(\bar{\Delta} \bar{a}^{k_{2}} \bar{b} \Delta\right)$ giving:

$$
(\star \bar{\Delta} b a b)\left(a^{i_{2}} b\right)\left(a^{i_{3}} b\right) \cdots\left(a^{i_{j}}\right) \Delta\left(\bar{\Delta} \bar{a}^{k_{2}} \bar{b} \Delta\right)
$$

and the product does not end with ' $\bar{b} \Delta$ ' if and only if $k_{2}=i_{j-1}$, in which case it ends with $\cdots a^{i_{j-1}} \Delta$. This continues inductively for each pair of elements from the center outwards and we see that we finally reach ' $\star$ ' if and only if the product is of the form shown in Equation (3).

The first word corresponding to this is a correctly encoded PCP sequence which equals ' $\star$ ' if and only if it corresponds to a correct solution word, completing the proof. Note that set $P$ has 2 times the number of elements as the set $P^{\prime}$. Since PCP was shown to be undecidable for 7 pairs of words in [13] (even with the first and last pairs used only once), Fixed Element PCP is undecidable for 14 pairs of words.

Let us consider a small example of the second word encoding for the sequence of words $1, i_{1}, i_{2}, m$. Following the above proof, we correctly obtain:

$$
(\star \bar{\Delta} b a b)\left(a^{i_{1}} b\right)\left(a^{i_{2}} b\right)\left(a^{m} b\right)\left(\bar{b} \bar{a}^{m} \bar{b} \Delta\right)\left(\bar{\Delta} \bar{a}^{i_{2}} \bar{b} \Delta\right)\left(\bar{\Delta} \bar{a}^{i_{1}} \bar{b} \Delta\right)(\bar{\Delta} \bar{a} \bar{b} \Delta)=\star .
$$

\section{Applications of FEPCP and Periodicity of Computations}

In this section we show our main results via applications of the Fixed Element PCP and the construction of periodic traces in counter automaton models.

\subsection{Any Diagonal Matrix Problem}

Problem 2. Any Diagonal Matrix Problem - Given a finite set of matrices $G$ generating a semigroup $S$. Does there exist any matrix $D \in S$ such that $D$ is a diagonal matrix?

This problem was considered for integral matrices in [6], but we shall now show that it is undecidable for rational complex matrix semigroups by using the Fixed Element PCP. In our proof we shall exhibit a semigroup that has no diagonal matrices if the instance of PCP has no solution and an infinite number of diagonal matrices (i.e. powers of a specific, known diagonal matrix) if the PCP instance does have a solution.

Theorem 2. Given a finitely generated matrix semigroup $S \subseteq \mathbb{C}(\mathbb{Q})^{4 \times 4}$, it is algorithmically undecidable to determine whether there exists any matrix $D \in S$ such that $D$ is a diagonal matrix.

Proof. We shall utilize the Fixed Element PCP (FEPCP) and the matrix representation $\zeta$ of a free group of rational quaternions. Let us define a function $\zeta:\{a, b, \bar{a}, \bar{b}\} \rightarrow \mathbb{C}(\mathbb{Q})^{2 \times 2}$ (where $\mathbb{C}(\mathbb{Q})$ denotes the field of complex numbers with rational coefficients):

$$
\begin{aligned}
& \zeta(a)=\left(\begin{array}{cc}
\frac{3}{5}+\frac{4}{5} \mathbf{i} & 0 \\
0 & \frac{3}{5}-\frac{4}{5} \mathbf{i}
\end{array}\right), \zeta(b)=\left(\begin{array}{cc}
\frac{3}{5} & \frac{4}{5} \\
-\frac{4}{5} & \frac{3}{5} \\
\frac{3}{5} & -\frac{4}{5} \\
\frac{4}{5} & \frac{3}{5}
\end{array}\right), \\
& \zeta(\bar{a})=\left(\begin{array}{cc}
\frac{3}{5}-\frac{4}{5} \mathbf{i} & 0 \\
0 & \frac{3}{5}+\frac{4}{5} \mathbf{i}
\end{array}\right), \zeta(\bar{b}) .
\end{aligned}
$$


and $\zeta$ forms a free group as proved in [4]. Recall also that in the Fixed Element PCP we have an alphabet of 7 letters, $\Gamma=\{a, \bar{a}, b, \bar{b}, \Delta, \bar{\Delta}, \star\}$. We shall use a homomorphism, $\gamma$, to encode these letters using elements of $\zeta$. Specifically, define $\gamma: \Gamma^{*} \rightarrow \mathbb{C}(\mathbb{Q})^{2 \times 2}$ by:

$$
\begin{aligned}
\gamma(\star)=\zeta(a), \gamma(a) & =\zeta(b a b), \gamma(b)=\zeta\left(b^{2} a^{2} b^{2}\right), \gamma(\Delta)=\zeta\left(b^{3} a^{3} b^{3}\right) \\
\gamma(\bar{a}) & =\zeta(\bar{b} \bar{a} \bar{b}), \gamma(\bar{b})=\zeta\left(\bar{b}^{2} \bar{a}^{2} \bar{b}^{2}\right), \gamma(\bar{\Delta})=\zeta\left(\bar{b}^{3} \bar{a}^{3} \bar{b}^{3}\right)
\end{aligned}
$$

and then extending to a monoid homomorphism in the usual way. Now, given an instance of FEPCP:

$$
P=\left\{\left(u_{1}, v_{1}\right),\left(u_{2}, v_{2}\right), \ldots,\left(u_{n}, v_{n}\right)\right\} \subseteq \Gamma^{*} \times \Gamma^{*},
$$

we shall use the mixed product property of Kronecker products that for any four matrices $A, B, C, D \in \mathbb{C}^{j \times j}:$

$$
(A B \otimes C D)=(A \otimes C)(B \otimes D) .
$$

We create a final set of 4-dimensional rational complex matrices:

$$
T=\left\{\gamma\left(u_{1}\right) \otimes \gamma\left(v_{1}\right), \gamma\left(u_{2}\right) \otimes \gamma\left(v_{2}\right), \ldots, \gamma\left(u_{n}\right) \otimes \gamma\left(v_{n}\right)\right\} \subseteq \mathbb{C}(\mathbb{Q})^{4 \times 4} .
$$

From the definition of FEPCP, we know a solution $w=w_{1} w_{2} \cdots w_{k}$ gives the equation $u_{w_{1}} u_{w_{2}} \cdots u_{w_{k}}=v_{w_{1}} v_{w_{2}} \cdots v_{w_{k}}=\star$ for the special symbol $\star$. Using our above encoding, we see that $\gamma(\star)=\zeta(a)$ which is clearly diagonal. Thus, given a correct solution to FEPCP, there exists a matrix $D \in\langle T\rangle$ such that:

$$
D=\gamma\left(u_{w_{1}} u_{w_{2}} \cdots u_{w_{k}}\right) \otimes \gamma\left(v_{w_{1}} v_{w_{2}} \cdots v_{w_{k}}\right)=\gamma(\star) \otimes \gamma(\star)=\zeta(a) \otimes \zeta(a),
$$

which is diagonal (since the Kronecker product of two diagonal matrices is diagonal). It can be seen that any word which is not a solution will contain at least one matrix $\zeta(b)$ or $\zeta(\bar{b})$ and since these are not diagonal, the tensor product will not be diagonal either.

\subsection{The Recurrent Matrix Problem}

The next problem that we consider here is the problem of whether an element of a matrix semigroup has an infinite number of factorizations over elements of the generator. This question is trivially undecidable in the case of singular matrices since it can be reduced to the mortality problem (whether a zero matrix belongs to a semigroup). Here we show that this problem is also undecidable for invertible matrix semigroups.

Problem 3. Recurrent Matrix Problem - Given a matrix $B$ and a semigroup $S$ generated by a finite set of matrices $G$. Does $B$ have an infinite number of factorizations over elements of $G$ ?

Theorem 3. The recurrent matrix problem is undecidable for non-singular $4 \times 4$ integer matrix semigroups.

Proof. From the construction in Proposition 4 we have that the problem of deciding if a nondeterministic two-counter automaton has an infinite number of trajectories leading to a final state $s_{\text {final }}$ with zero counters is undecidable. We can encode and simulate this automaton 
by PCP, as shown in Proposition 2. We shall now also reduce it to the FEPCP which will be directly applicable to the recurrent matrix problem.

Thus, if the instance of FEPCP has a solution then a nondeterministic two-counter automaton has an infinite number of trajectories leading to a final state $s_{\text {final }}$ with zero counters and FEPCP also has an infinite number of different sequences of indices that will lead to the solution $(\star, \star)$. We now show how to encode an instance of FEPCP into a matrix semigroup.

Let $\Sigma=\{a, b\}$ be a binary alphabet and $\bar{\Sigma}=\{\bar{a}, \bar{b}\}$ be the inverse elements of $\Sigma$, i.e., $\bar{a}=a^{-1}$ and $\bar{b}=b^{-1}$. We define the homomorphism $\varphi:(\Sigma \cup \bar{\Sigma})^{*} \rightarrow \mathbb{Z}^{2 \times 2}$ by:

$$
\varphi(a)=\left(\begin{array}{ll}
1 & 2 \\
0 & 1
\end{array}\right), \varphi(b)=\left(\begin{array}{ll}
1 & 0 \\
2 & 1
\end{array}\right), \varphi(\bar{a})=\left(\begin{array}{cc}
1 & -2 \\
0 & 1
\end{array}\right), \varphi(\bar{b})=\left(\begin{array}{cc}
1 & 0 \\
-2 & 1
\end{array}\right)
$$

It is well known that $\varphi$ forms a free group of $2 \times 2$ matrices from the free group of words generated by $\Sigma \cup \bar{\Sigma}$.

Let $\Gamma=\{a, \bar{a}, b, \bar{b}, \Delta, \bar{\Delta}, \star\}$ and define a new mapping $\gamma$, to encode $\Gamma$ using elements of $\varphi$, where $\gamma: \Gamma^{*} \rightarrow \mathbb{Z}^{2 \times 2}$ is given by:

$$
\begin{aligned}
\gamma(\star)=\varphi(a), \gamma(a) & =\varphi(b a b), \gamma(b)=\varphi\left(b^{2} a^{2} b^{2}\right), \gamma(\Delta)=\varphi\left(b^{3} a^{3} b^{3}\right) \\
\gamma(\bar{a}) & =\varphi(\bar{b} \bar{a} \bar{b}), \gamma(\bar{b})=\varphi\left(\bar{b}^{2} \bar{a}^{2} \bar{b}^{2}\right), \gamma(\bar{\Delta})=\varphi\left(\bar{b}^{3} \bar{a}^{3} \bar{b}^{3}\right)
\end{aligned}
$$

and then extending to a monoid homomorphism. Given an instance of FEPCP $P=\left\{\left(u_{i}, v_{i}\right) \mid 1 \leq\right.$ $i \leq n\}$, for each $1 \leq i \leq n$ we define the following matrices:

$$
A_{i}=\left(\begin{array}{cc}
\gamma\left(u_{i}\right) & 0 \\
0 & \gamma\left(v_{i}\right)
\end{array}\right)
$$

If the matrix

$$
B=\left(\begin{array}{cc}
\gamma(\star) & 0 \\
0 & \gamma(\star)
\end{array}\right)
$$

belongs to the semigroup $S$ generated by $G=\left\{A_{1}, \ldots, A_{n}\right\} \subseteq \mathbb{Z}^{4 \times 4}$ then FEPCP has a solution. According to the construction of the automaton, the FEPCP has a solution if the automaton halts and has an infinite number of trajectories leading to its final state. It also follows that the number of different paths leading to $B$ is infinite. Since the existence of an infinite number of trajectories leading to the final state is an undecidable problem then the recurrent matrix problem is also undecidable.

\subsection{The Vector Ambiguity Problem}

Problem 4. Vector Ambiguity Problem - Given a semigroup $S$ of $n \times n$ matrices and an initial $n$-dimensional vector $u$. Let $V$ be a set of vectors such that $V=\{v: v=M u ; M \in S\}$. Do $S$ and $u$ generate a non-repetitive set of vectors? In other words the question is whether for every vector $v$ of set $V$ there is a unique matrix $M \in S$ such that $M \cdot u=v$ ?

Theorem 4. The vector ambiguity problem is undecidable for matrix semigroups over integers in dimension 4 and over rationals in dimension 3. 
Proof. Let $M$ be a two-counter machine that has no halting configuration. The problem of determining if $\mathrm{M}$ has a periodic configuration is undecidable. Let us use a construction proposed in Proposition 2, which simulates any two-counter machine by a set of pairs of words. Note that our method does not define a halting state of the machine and in this case we only predefine an initial configuration of a counter machine $M$.

Assume that the set of pairs of words used to simulate a counter machine is $P=\left\{\left(u_{i}, v_{i}\right) \mid 1 \leq\right.$ $i \leq n\}$. Let us construct a set of pairs of $2 \times 2$ matrices using the homomorphism $\varphi$ i.e. $\left\{\left(\varphi\left(\overline{u_{1}}\right), \varphi\left(v_{1}\right)\right), \ldots,\left(\varphi\left(\overline{u_{n}}\right), \varphi\left(v_{n}\right)\right)\right\}$.

Instead of equation $u=v$ we consider a concatenation of two words $\bar{u} \cdot v$ which equals $\varepsilon$ only in the case where $u=v$. We associate $2 \times 2$ matrix $C$ with a word $w$ of the form $u \cdot \bar{v}$. Initially $C$ is a matrix that corresponds to the initial configuration of the machine which is stored in the first pair $\left(u_{1}, v_{1}\right)$, so $C=\varphi\left(\overline{u_{1}}\right) \cdot \varphi\left(v_{1}\right)$.

The extension of a word $w$ by a new pair of words $\left(\overline{u_{r}}, v_{r}\right)$ (i.e., that gives us $w^{\prime}=\overline{u_{r}} \cdot w \cdot v_{r}$ ) corresponds to the following matrix multiplication

$$
C_{w^{\prime}}=\varphi\left(\overline{u_{r}}\right) \cdot C_{w} \cdot \varphi\left(v_{r}\right)
$$

Let us rewrite operation (4) in more detail.

$$
\left(\begin{array}{ll}
c_{w^{\prime}}^{11} & c_{w^{\prime}}^{12} \\
c_{w^{\prime}}^{21} & c_{w^{\prime}}^{22}
\end{array}\right)=\left(\begin{array}{ll}
u^{11} & u^{12} \\
u^{21} & u^{22}
\end{array}\right) \cdot\left(\begin{array}{ll}
c_{w}^{11} & c_{w}^{12} \\
c_{w}^{21} & c_{w}^{22}
\end{array}\right) \cdot\left(\begin{array}{ll}
v^{11} & v^{12} \\
v^{21} & v^{22}
\end{array}\right)
$$

In this case, pairwise multiplication will correspond to an update of the current state according to the operation of a two-counter machine $M$.

Let us consider the dynamics of changes for the matrix $C$. It is easy to see that in the case of an incorrectly applied command for a machine $M$, the pairwise concatenation (multiplication) will lead to increase of the length for a word $w$ and will never end up in a repeated word after that. Therefore after an incorrect application of a command of $M$, a matrix $C$ will never have the same value again. The correct application of pairwise concatenation of words or multiplication of matrices covers the set of correct configurations of a two-counter machine $M$. In the case of a periodic two-counter machine, the finiteness of the configuration space will lead to the finiteness of the set $X$ of possible $C$ matrices that can be generated during the correct application of rules for $M$, since every matrix $C \in X$ corresponds to a unique reachable configuration of $M$. Thus the set of matrices that can be generated by pairwise multiplication may contain repetitions if and only if the two-counter machine has periodic behavior.

In order to finish the proof of undecidability for the case of an integer matrix semigroup, we represent matrix $C$ as a vector $x=\left(c_{w}{ }^{11}, c_{w}{ }^{12}, c_{w}{ }^{21}, c_{w}{ }^{22}\right)^{T}$ increasing the dimension to 4 and rewriting pairwise multiplication as a 4-dimensional linear transformation of vector $x$.

$$
\left(\begin{array}{l}
c_{w^{\prime}}^{11} \\
c_{w^{\prime}}^{12} \\
c_{w^{\prime}}^{21} \\
c_{w^{\prime}}^{22}
\end{array}\right)=\left(\begin{array}{ll}
u^{11} & u^{12} \\
u^{21} & u^{22}
\end{array}\right) \otimes\left(\begin{array}{ll}
v^{11} & v^{12} \\
v^{21} & v^{22}
\end{array}\right)^{T} \cdot\left(\begin{array}{l}
c_{w}^{11} \\
c_{w}^{12} \\
c_{w}^{21} \\
c_{w}^{22}
\end{array}\right)
$$

The same method can be used to prove the undecidability of the vector ambiguity problem in dimension three for rational matrices by instead of using $\varphi$, using another homomorphism $\psi$ based on a free group:

$$
\psi(a)=\left(\begin{array}{ll}
1 & 1 \\
0 & 2
\end{array}\right) \quad \psi(b)=\left(\begin{array}{ll}
1 & 2 \\
0 & 2
\end{array}\right) \quad \psi(\bar{a})=\left(\begin{array}{cc}
1 & -\frac{1}{2} \\
0 & \frac{1}{2}
\end{array}\right) \quad \psi(\bar{b})=\left(\begin{array}{cc}
1 & -1 \\
0 & \frac{1}{2}
\end{array}\right)
$$


Since we multiply only upper triangular matrices in $(5)$ we have that $c_{w}^{21}$ is equal to zero in the initial moment and after every next multiplication. When we rewrite (5) into a 4dimensional linear transformation as is shown in (6), the third row and the third column can be removed from the $4 \times 4$ matrix in (6). Finally, we rewrite (6) as a 3 -dimensional linear transformation for the vector $\left(c_{w}{ }^{11}, c_{w}{ }^{12}, c_{w}{ }^{22}\right)^{T}$.

Note that from the above result it also follows that it is undecidable whether there exists a periodic trace of configurations in a one state blind nondeterministic 4-counter machine with only counter updates in terms of linear transformations.

\subsection{The Infinite Post Correspondence Problem}

As another application of periodic counter machines we state a new variant of the infinite PCP. It was shown in [5] that the infinite Post correspondence problem is undecidable for 105 pairs of words. This result was later improved to 9 by V. Halava and T. Harju [9] by encoding semi-Thue systems and utilizing Claus's construction for PCP. The authors of [9] also show a related result that determining if a particular PCP instance has a solution that is non-ultimately periodic is undecidable.

Lemma 1. [9] If the termination problem is undecidable for $n$-rule semi-Thue systems, then it is undecidable for instances of the PCP size $n+3$ whether or not there exists an infinite solution that is not ultimately periodic.

We recall that an infinite word is said to be ultimately periodic if it can be written in the form $w=u v^{\infty}$ where $u, v$ are non-empty, finite words.

Using an encoding of two-counter machines into pairs of words and thus PCP instances and Proposition 3 (shown in [7]), we can derive a related result.

Lemma 2. There exists a class of instances of Post's correspondence problem which have a guaranteed single infinite solution and no finite solution where it is undecidable whether the solution is ultimately periodic.

Proof. We use the construction from Proposition 2 which allows us to simulate an arbitrary two-counter machine by a finite set of pairs of words as is done in Post's correspondence problem. We shall use the idea from Proposition 3 in which we start with an initial counter machine $M$ and create a second counter machine $M^{\prime}$ such that $M^{\prime}$ does not halt and $M^{\prime}$ has a periodic configuration if and only if $M$ halts on its input in the same way as was originally done in [7]. Since determining if an arbitrary counter machine $M$ halts is undecidable, determining if $M^{\prime}$ has a periodic configuration is undecidable as explained in Proposition 3.

It is well known that a $k$-counter machine can be simulated by a two-counter machine. Therefore, from machine $M^{\prime}$, we create a third machine $M^{\prime \prime}$ which has only two counters. Using the construction from Proposition 2, we can simulate $M^{\prime \prime}$ via an instance of PCP which we denote by $P$. However, due to the construction of $M^{\prime}$, we know it does not halt, thus $P$ has no solution.

The counter machine $M^{\prime}$ is deterministic and has a guaranteed infinite run. Therefore instance $P$ has a single infinite word solution. By the conversion from a two-counter machine 
to a PCP instance from Proposition 2, there is a single letter $\gamma_{1} \in \Gamma$ which must be used first and then never again. Therefore, the infinite solution of PCP is of the form:

$$
w^{\prime}=\gamma_{1} w ; \quad \gamma_{1} \in \Gamma, w \in\left(\Gamma \backslash\left\{\gamma_{1}\right\}\right)^{\infty} .
$$

Since determining if $M^{\prime}$ is periodic is undecidable, it is also undecidable whether $w$ is a periodic word, or equivalently, whether $w^{\prime}$ is ultimately periodic, thus completing the proof.

\section{Acknowledgements}

We would like to thank the referees for their careful checking of this manuscript, especially the referee who noticed a gap in one of our earlier proofs.

\section{References}

1. P. A. Abdulla, B. Jonsson, Undecidable Verification Problems for Programs with Unreliable Channels. Inf. Comput. 130(1),(1996) 71-90.

2. L. Babai, R. Beals, J. Cai, G. Ivanyos, E. M. Luks, Multiplicative Equations Over Commuting Matrices. ACM-SIAM Symposium on Discrete Algorithms, (1996), 28-30.

3. P. Bell and I. Potapov, Lowering Undecidability Bounds for Decision Questions in Matrices. Developments in Language Theory, DLT06, LNCS 4036, (2006), 375-385.

4. P. Bell, I. Potapov, Reachability Problems in Quaternion Matrix and Rotation Semigroups. MFCS 2007, 346-358.

5. V. Blondel, V. Canterini, Undecidable Problems for Probabilistic Automata of Fixed Dimensions, Theory Comput. Syst., 36(3), (2003), 231-245.

6. V. Blondel, J. Cassaigne, J. Karhumäki, Problem 10.3. Freeness of Multiplicative Matrix Semigroups, Unsolved Problems in Mathematical Systems and Control Theory, Edited by V.Blondel and A.Megretski, 309-314.

7. V. Blondel, J. Cassaigne, C. Nichitiu, On the Presence of Periodic Configurations in Turing Machines and in Counter Machines, Theoretical Computer Science, 289, (2002), 573-590.

8. V. Halava, T. Harju, M. Hirvensalo, Undecidability Bounds for Integer Matrices using Claus Instances, TUCS Technical Report No. 766, (2006).

9. V. Halava and T. Harju, Undecidability of Infinite Post Correspondence Problem for Instances of Size 9, Theoretical Informatics and Applications, 40, (2006), 551-557.

10. I. Korec, Small Universal Register Machines, Theoretical Computer Science, 168, (1996), 267-301.

11. O. Kurganskyy, I. Potapov. Computation in One-Dimensional Piecewise Maps and Planar Pseudo-Billiard Systems. Unconventional Computation (2005), 169-175.

12. A. Lisitsa, I. Potapov. In Time Alone: On the Computational Power of Querying the History, TIME'06, (2006), 42-49.

13. Y. Matiyasevic, G. Sénizergues, Decision Problems for Semi-Thue Systems with a Few Rules, Theoretical Computer Science, 330, (2005), 145-169.

14. M. Minsky, Recursive Unsolvability of Post's Problem of "tag" and other Topics in Theory of Turing Machines, Annals of Mathematics, 74, (1961), 437-455.

15. M. Minsky, Computation: Finite and Infinite Machines, Prentice-Hall International, (1967).

16. I. Potapov. From Post Systems to the Reachability Problems for Matrix Semigroups and Multicounter Automata. Developments in Language Theory, LNCS 3340, (2004), 345-356.

17. M. Sipser, Introduction to the Theory of Computation, PWS Publishing, (1997). 